\title{
Detection of Unicolor ECG Electrode Reversals in Standard 12-Lead ECG
}

\author{
Irena Jekova ${ }^{1}$, Remo Leber ${ }^{2}$, Vessela Krasteva ${ }^{1}$, Ramun Schmid ${ }^{2}$ \\ ${ }^{1}$ Institute of Biophysics and Biomedical Engineering, Bulgarian Academy of Sciences, Sofia, Bulgaria \\ ${ }^{2}$ Signal Processing, Schiller AG, Baar, Switzerland
}

\begin{abstract}
This paper presents the performance of a commercial lead quality monitoring library (LQMLib, Schiller AG) for detection of reversals between 12-lead ECG cables with matching colors and proposes methods for improvements, where necessary. The study is performed on a large 12-lead ECG database with 1331 chest pain patients (646 training, 685 test recordings) and ECGs from 29 volunteers with swapped red, yellow, green, black and all unicolor cables. Relying on assessment of inter-lead correlations over continuous $4 \mathrm{~s}$ episodes, LQMLib without adjustments achieves Se $>97 \%$ for all unicolor cable reversals, except RC1 $(\mathrm{Se}=29 \%)$ and NC5 (Se $=88 \%)$. The proposed additional analyses improve RC1 and NC5 swap detection by 63\% points and 7\% points, respectively.
\end{abstract}

\section{Introduction}

The correct electrode placement is a crucial factor for proper electrocardiogram (ECG) interpretation. Cable reversals have been observed in 0.4-4 \% of 12-lead ECG recordings [1] that could confuse physicians and provoke unnecessary diagnostic tests and patient treatment.

Numerous approaches were proposed for the automated detection of limb ECG electrode reversals:

- Left arm (L) and left leg (F) reversal was examined by $\mathrm{P}$ wave amplitude [2] and QRS, P-axes [3];

- Right arm (R) and right leg (N) reversal was detected by observing flat line ECG in lead II [4];

- Various L/R/F/N reversals were detected using the direction of P-loop inscription and/or frontal P-axis [5]; frontal QRS-axis [6]; lead reconstruction using redundancy of information in 8 independent leads [7]; morphological measurements of QRS, P-wave amplitudes, frontal axis and clockwise vector loop rotation, combined with redundancy features [8]; maximal and minimal QRS, T-wave voltages in leads I, II, III [9]; correlation coefficients, comparing limb leads to V6 [10]; gathering the features described in [3] and [7] for a more robust and accurate performance [11].
Fewer studies proposed solutions for detection of chest lead reversals. Among the mentioned publications for limb leads, only four addressed chest leads reversals:

- 5 reversals of adjacent leads (V1/V2, V2/V3, V3/V4, $\mathrm{V} 4 / \mathrm{V} 5$ and V5/V6) were analysed in [3];

- 9 reversals, including 5 adjacent leads, V1/V3, V4/V6, $\mathrm{V} 4 / \mathrm{V} 5 / \mathrm{V} 6 / \mathrm{V} 1 / \mathrm{V} 2 / \mathrm{V} 3$ and V6/V5/V4/V3/V2/V1 were evaluated in [7];

- 7 reversals, including 5 adjacent leads, V1/V3 and V4/V6 were handled in [8];

- 15 reversals, including all possible pairwise V1-V6 swaps were tested in our previous study [10].

Recently, Joshi et al [12] presented an interesting case of a chest pain patient with an initial ECG demonstrating ST-segment elevation in the inferior leads, whereas the subsequent ECG showed ST elevation in the chest leads. The authors postulated that this "wandering myocardial infarction" resulted from an interchange of the chest lead and limb lead cables of the ECG machine. Their thesis was impugned by Givens et al [13], who explained the wandering ST elevation with medical reasons. Such a dispute arises the need for advanced detectors of reversals between limb and chest leads.

Although the interchange between 12-lead ECG cables with matching colors is quite possible scenario, the effect of this problem has not been addressed in the literature so far. The aim of this study is to test the ability of a commercial lead quality monitoring library (LQMLib, Schiller AG) to detect reversals between limb and chest unicolor ECG electrodes (IEC color coding), and to propose methods for improvements, where necessary.

\section{ECG databases}

This study used two 10s resting 12-lead ECG databases collected under the following conditions:

- DB1: Clinical examination of 1331 chest pain patients at the Emergency Department of the Basel University Hospital. Standard 12-lead ECGs were acquired via the commercial Schiller CS-200 Excellence. The device did not give feedback with respect to lead reversals, so we assumed correct lead placement during the examination. Further, lead reversals were retrospectively simulated and their detection performance 
was tested on 685 ECGs from DB1 (Test) and trained on other 646 ECGs from DB1 (Train), when necessary.

- DB2: Test bench examination of 29 non-cardiac volunteers by recording of 12-lead ECG via 10electrode cable with IEC color coding standard, applying prospective electrode cable reversals at the time of the recording. Six recordings per subject were collected, including correct position of the electrodes, swaps of red (RC1), yellow (LC2), green (FC3), black (NC5) electrodes, as well as swap of all unicolor electrodes (RC1-LC2-FC3-NC5).

- All signals were recorded at $1 \mathrm{kHz}$ sampling rate, $1 \mu \mathrm{V}$ resolution, pre-filtered in a bandwidth (0.5-25) Hz.

\section{Methods}

This section addresses the method for simulation of unicolor electrode reversals, as well as the new methods for improving the detection of two unicolor lead reversals (RC1, NC5). They have been proposed in response to the observed performance drop (Table 1), considering that the basic LQMLib algorithm [10] has not been designed to detect any interchanges between limb and chest electrodes, including unicolor ones.

\subsection{Simulation of lead reversals}

The reversals between red, yellow and green electrodes are simulated by the following transform:

$$
\left[\begin{array}{l}
\text { Irev } \\
\text { Irev } \\
V_{X} \text { rev }
\end{array}\right]=\mathbf{M S M}_{\mathrm{inv}}\left[\begin{array}{c}
I \\
I I \\
V_{X}
\end{array}\right]
$$

where $I, I I, V_{X}$ are the correct ECG leads; Irev, IIrev, $V_{X} r e v$ are the ECG leads after reversals; $M$ is the matrix that converts the potentials of the limb $(\mathrm{R}, \mathrm{L}, \mathrm{F})$ and chest electrodes $\left(C_{X}\right)$ into leads I, II and $V_{X}, M_{\text {inv }}$ does the opposite and $\mathrm{S}$ is the matrix that swaps the electrodes.

The reversal between black electrodes (NC5) is approximated by setting $\mathrm{C} 5$ potential equal to $\mathrm{F}(\mathrm{C} 5 \approx \mathrm{F})$.

\subsection{RC1 reversal detection}

The LQMLib algorithm for detection of chest lead reversals is based on the inter-lead ECG waveform similarities within $4 \mathrm{~s}$, following the normal progression of the cardiac vector projection on the horizontal plane (V1-V6) [10]. It estimates a square matrix $\operatorname{rLS}(6 \times 6)$, containing the inter-lead correlation coefficients between all pairwise combinations of leads within the standard set: LS = [V1, V2, V3, V4, V5, V6] - Figure 1.

The new method for detection of RC1 reversal assumes the neighbouring positions of R, C1 electrodes on the human body. It extends rLS beyond V1, including lead $\mathrm{VR}=2 * \mathrm{aVR} / 3$ as the right arm potential vs. Wilson Central Terminal (WCT).

\begin{tabular}{|c|c|c|c|c|c|c|c|}
\hline & VR & V1 & V2 & V3 & V4 & V5 & V6 \\
\hline VR & 1 & $>$ & $>$ & $>$ & $>$ & $>$ & $>$ \\
\hline V1 & $<$ & 1 & $>$ & $>$ & $>$ & $>$ & $>$ \\
\hline V2 & $<$ & $<$ & 1 & $>$ & $>$ & $>$ & $>$ \\
\hline V3 & $<$ & $<$ & $<$ & 1 & $>$ & $>$ & $>$ \\
\hline V4 & $<$ & $<$ & $<$ & $<$ & 1 & $>$ & $>$ \\
\hline V5 & $<$ & $<$ & $<$ & $<$ & $<$ & 1 & $>$ \\
\hline V6 & $<$ & $<$ & $<$ & $<$ & $<$ & $<$ & 1 \\
\hline
\end{tabular}

Figure 1. Extension of square LQMLib correlation matrix (blue rectangle V1-V6) with lead VR for detection of $\mathrm{RC} 1$ reversal. The color gradient shows the extension of LSE matrices from [VR,V1-V2] to [VR,V1-V6] during training. The signs $\left({ }^{\prime}<\right.$ ', ' $\left.>^{\prime}\right)$ show the normal decrease of the correlation coefficients rightwards and leftwards from the diagonal (' 1 ') when the inter-lead distance increases.

As shown in figure 1, LS extensions with different sizes around [VR, V1, V2] are applicable and further trained:

- LSE3: 3 leads [VR, V1, V2];

- LSE4: 4 leads [VR, V1, V2, V3];

- LSE5: 5 leads [VR, V1, V2, V3, V4];

- LSE6: 6 leads [VR, V1, V2, V3, V4, V5];

- LSE7: 7 leads [VR, V1, V2, V3, V4, V5, V6].

\subsection{NC5 reversal detection}

The proposed method for NC5 reversal detection relies on the assumption for similar potentials at both legs $(\mathrm{N} \approx \mathrm{F})$, applying two concurrent criteria:

- $\mathrm{VD}_{\mathrm{pp}}<\mathrm{THR}$ : Low peak-to-peak amplitude difference between $\mathrm{F}$ and $\mathrm{C} 5$ potentials $\left(\mathrm{VD}_{\mathrm{pp}}=|\mathrm{V} 5-\mathrm{VF}|\right)$, where $\mathrm{VF}=2 * \mathrm{aVF} / 3$ is the left leg potential vs. WCT.

- relVD $\mathrm{pp}_{\mathrm{pp}}=$ mean $(|\mathrm{V} 4-\mathrm{VF}|,|\mathrm{V} 6-\mathrm{VF}|) / \mathrm{VD}_{\mathrm{pp}}>\mathrm{K}:$ relVD $\mathrm{pp}_{\mathrm{pp}}$ values tending to unity are a sign for $\mathrm{C5}$ potential comparable to $\mathrm{C} 4$, C6, when the decision for NC5 reversal is not relevant.

The peak-to-peak amplitudes of $\mathrm{VD}_{\mathrm{pp}}$, relVD $\mathrm{Dp}_{\mathrm{pp}}$ are assessed in non-overlapping $1 \mathrm{~s}$ intervals, and the median values over $4 \mathrm{~s}$ are adopted in the above criteria for improving the noise immunity.

\section{Results and discussion}

LQMLib (without adjustments) is tested on DB1 (Test) after simulation of all 15 possible unicolor electrode reversals. Table 1 shows specificity $\mathrm{Sp}=92.2 \%$ and sensitivity Se $>97.3 \%$ for all reversals, except:

- RC1 (Se=29.1\%): Due to the neighbouring position of R-C1 in the right part of the human body, their reversal does not change the gradual progression of the ECG waveform in the swapped rLS matrix [VR,V2-V6].

- NC5 (Se=88\%): In occasional cases, the waveforms of V5 (lateral) and VF (inferior) look highly correlated, thus not interrupting the normal progression of ECG waveform in the swapped rLS matrix [V1-V4,VF,V6]. 
Table 1. Test performance of LQMLib for detection of simulated electrode reversals over DB1 (Test). LQMLib has not been adjusted to any of the tested reversals.

\begin{tabular}{ll}
\hline Lead reversal & Performance \\
\hline Correct electrode placement & $\mathrm{Sp}=92.2 \%$ \\
\hline RC1 (red) & $\mathrm{Se}=29.1 \%$ \\
LC2 (yellow) & $\mathrm{Se}=97.3 \%$ \\
FC3 (green) & $\mathrm{Se}=97.5 \%$ \\
NC5 (black) & $\mathrm{Se}=88.0 \%$ \\
\hline RC1-LC2 (red/yellow) & $\mathrm{Se}=98.5 \%$ \\
RC1-FC3 (red/green) & $\mathrm{Se}=99.2 \%$ \\
RC1-NC5 (red/black) & $\mathrm{Se}=92.2 \%$ \\
LC2-FC3 (yellow/green) & $\mathrm{Se}=99.8 \%$ \\
LC2-NC5 (yellow/black) & $\mathrm{Se}=100 \%$ \\
FC3-NC5 (green/black) & $\mathrm{Se}=100 \%$ \\
\hline RC1-LC2-FC3 (red/yellow/green) & $\mathrm{Se}=100 \%$ \\
RC1-LC2-NC5 (red/yellow/black) & $\mathrm{Se}=100 \%$ \\
RC1-FC3-NC5 (red/green/black) & $\mathrm{Se}=100 \%$ \\
LC2-FC3-NC5 (yellow/green/black) & $\mathrm{Se}=100 \%$ \\
\hline RC1-LC2-FC3-NC5 (all colors) & $\mathrm{Se}=100 \%$ \\
\hline
\end{tabular}
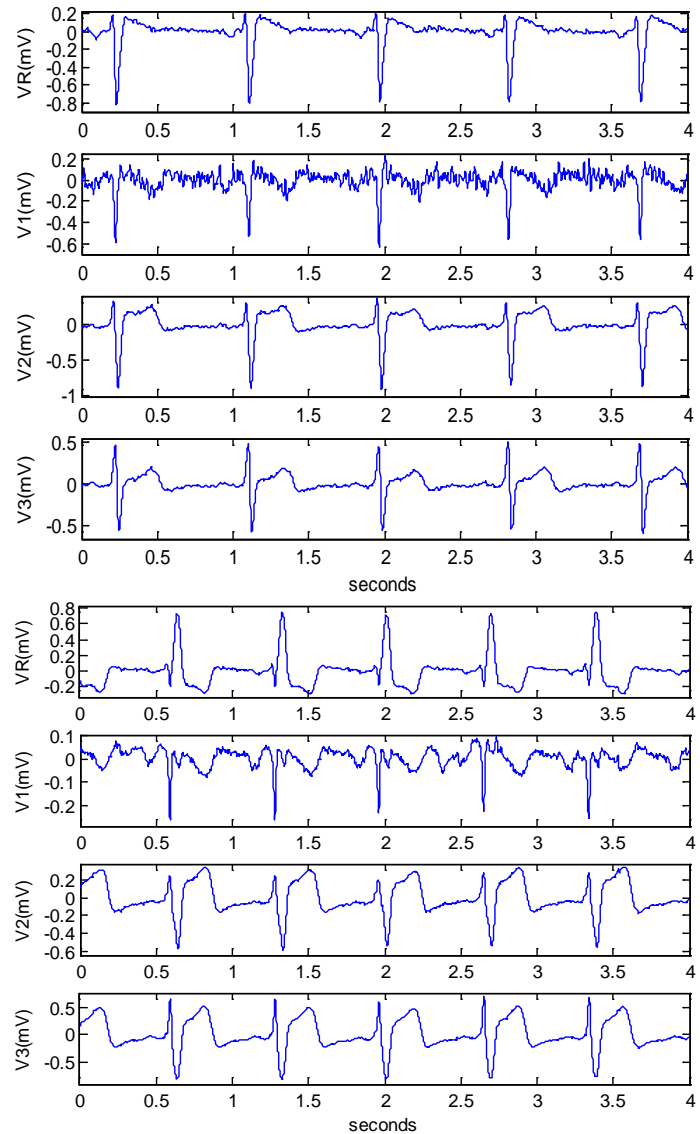

Figure 2. Examples with simulated RC1 reversals:

(a) True positive (TP) detection by LSE4, sensitive to interruption of Q-wave increase in V1;

(b) False negative (FN) detection by LSE4 due to stronger correlation between V1,V2 compared to VR, V2.
Table 2. Training performance of the new method for detection of RC1 reversal over DB1(Train), applying extended LS matrices with different sizes. The optimal result is highlighted for LSE4 [VR, V1, V2, V3].

\begin{tabular}{llllll}
\hline Lead set & LSE3 & LSE4 & LSE5 & LSE6 & LSE7 \\
\hline Se (\%) & 90.2 & $\mathbf{9 1 . 6}$ & 90.6 & 87.3 & 81.4 \\
Sp (\%) & 98.9 & $\mathbf{9 8 . 5}$ & 98.0 & 97.4 & 96.7 \\
\hline
\end{tabular}

The choice of the best lead set is done by looking at the performance of different LS on DB1 (Train) - Table 2. Thus, LSE4 [VR, V1, V2, V3] is selected, providing max $\mathrm{Se}=91.6 \%$ for detection of the simulated RC1 reversal and high $\mathrm{Sp}=98.5 \%$ for detection of the correct electrode placement. Examples of LSE4 are presented in figures 2,3. Substantial Se drop (87-81\%) is observed for LSE6 and LSE7, scanning the ECG progression in V5, V6 - the most distant chest leads from the detected RC1 swap. LQMLib extended by LSE4 setting is tested on DB1 (Test), improving RC1 swap detection from $\mathrm{Se}=29.1 \%$ to 92.6\%, while keeping high $\mathrm{Sp}=92.2 \%$.
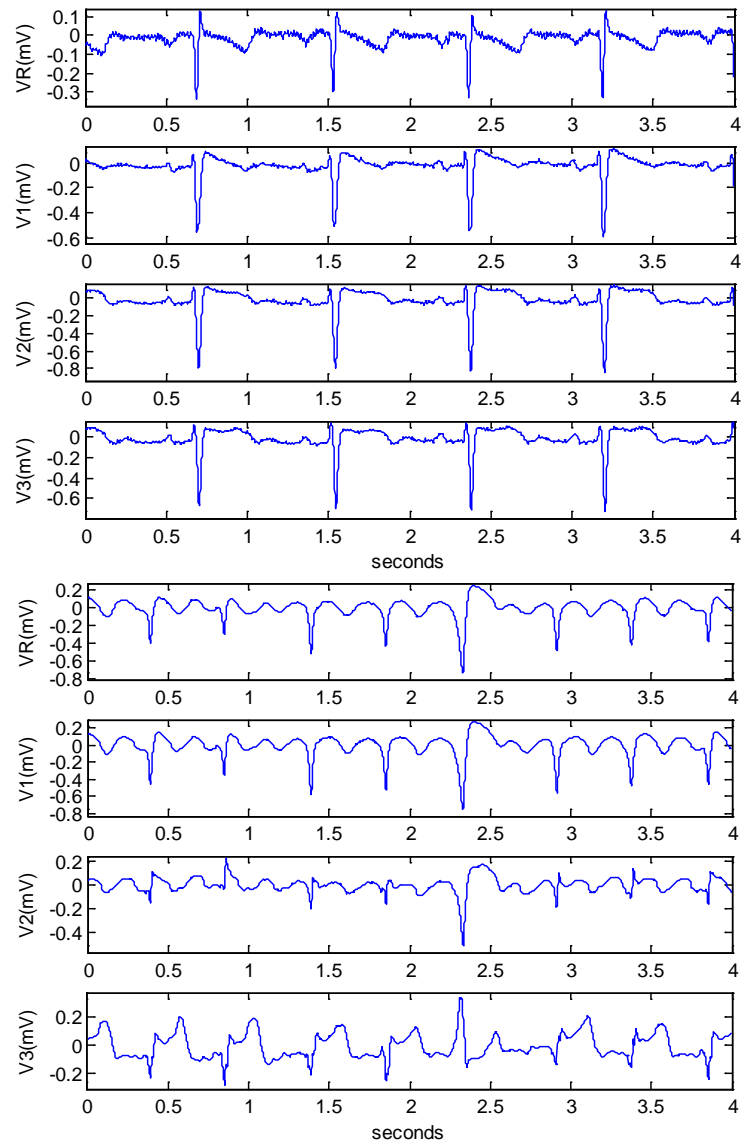

Figure 3. Examples with correct electrodes placement:

(a) True negative (TN) detection by LSE4, following expected gradual $\mathrm{PQRST}$ progression;

(b) False positive (FP) detection by LSE4 due to similar waveforms in VR and V1. 
The proposed amplitude criteria for detection of NC5 reversal are optimized on DB2 (Se, Sp) and DB1 (Train) (only Sp). DB1 could not be used for Se adjustment due to the equivalence between the first criterion for detection of NC5 reversal and the assumption $(\mathrm{C} 5 \approx \mathrm{F})$ in the simulation of black electrodes reversal (section 3.1), therefore unable to report Se.

The settings of the threshold values THR and $\mathrm{K}$ for detection of NC5 reversal are shown in Figure 4:

- $\quad \mathrm{THR}=150 \mu \mathrm{V}$ is set below VDpp observed for all recordings in DB2 and $>99 \%$ of the recordings in DB1 (Train) with correct electrode placement.

- $\mathrm{K}=2.2$ is set at $\left\{\right.$ mean(|V4-VF|,|V6-VF|)/VD $/ \mathrm{Vp}_{\mathrm{pp}}$ $\rightarrow$ min $\}$ among all recordings in DB2 with NC5 reversal, for which $\mathrm{VD}_{\mathrm{pp}}<150 \mu \mathrm{V}$

The designed amplitude criteria for NC5 reversal detection provide $\mathrm{Se}=59 \%$, keeping $\mathrm{Sp}=100 \%$ on $\mathrm{DB} 2$ and DB1(Train) - Table 3. Despite the limited Se, extending LQMLib with the NC5 criteria leads to Se improvement by $7 \%$ points ( $86 \%$ vs. $93 \%$ ) without Sp degradation on the training datasets. Considering the insignificant difference between Se of LQMLib on DB1(Test) and DB2 (88.0\% vs. 86.2\%, p=0.77), we could expect $\sim 7 \%$ point Se improvement in clinical conditions similar to the recording of DB1(Test). The analysis over DB1(Test) shows a negligible Sp drop of $0.3 \%$.

Table 3. Training/test performance of NC5 amplitude criteria run alone and together with LQMLib. LQMLib independent performance is provided for comparison.

\begin{tabular}{llllllll}
\hline & DB2 & & \multicolumn{2}{c}{ DB1(Train) } & \multicolumn{2}{l}{ DB1(Test) } \\
Method & Se(\%) & Sp(\%) & Se(\%) & Sp(\%) & Se(\%) & Sp(\%) \\
\hline NC5 crit & 58.6 & 100 & - & 100 & - & 99.6 \\
\hline LQMLib & 86.2 & 89.7 & 82.1 & 91.5 & 88.0 & 92.2 \\
\hline Combined 93.1 & 89.7 & - & 91.5 & - & 91.9 \\
\hline
\end{tabular}

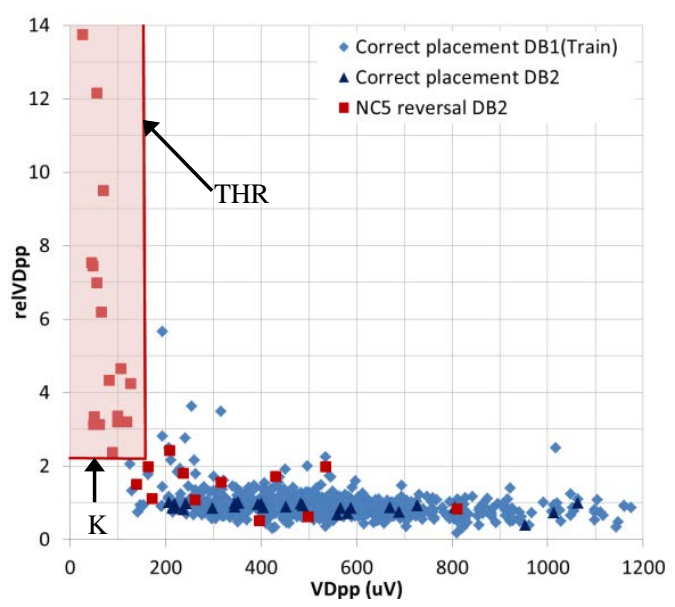

Figure 4. Scatterplot of the amplitude criteria $V D_{p p}$, relVD $D_{\mathrm{pp}}$ for ECG signals with correct electrode placement and NC5 reversal. The red area encloses cases that trigger the NC5 reversal detection.

\section{Conclusions}

LQMLib without adjustment provides Se $>97 \%$ for all unicolor cable reversals except RC1 ( $\mathrm{Se}=29 \%)$ and NC5 $(\mathrm{Se}=88 \%)$. We proposed additional analysis that improves RC1 swap detection ( $\mathrm{Se}=92.6 \%)$ and amplitude criteria, which could be useful for NC5 interchange recognition.

\section{References}

[1] Rudiger A, Hellermann J, Mukherjee R, Follath F, Turina J. Electrocardiographic artifacts due to electrode misplacement and their frequency in different clinical settings. Am J Emer Med 2007;25:174-8.

[2] Abdollah H,Milliken J. Recognition of electrocardiographic left arm/left leg lead reversal. Am J Cardiol 1997;80:1247-9

[3] Hedén B, Ohlsson M, Holst $H$, Mjöman M, Rittner R, Pahlm O, et al. Detection of frequently overlooked electrocardiographic lead reversals using artificial neural networks. Am J Cardiol 1996;78:600-4.

[4] Hoffman I. A flatline electrocardiogram in lead II is a marker for right arm/right leg electrode switch. J Electrocardiol 2007;40:226-7.

[5] Ho KKL, Ho SK. Use of the sinus $\mathrm{P}$ wave in diagnosing electrocardiographic limb lead misplacement not involving the right leg electrode. J Electrocardiol 2001;34:161-71.

[6] Ho RT, Mukherji L, Evans TJ. Simple diagnosis of limblead reversals by predictable changes in QRS axis. Pacing Clin Electrophysiol 2006;29:272-7.

[7] Kors JA, van Herpen G. Accurate automatic detection of electrode interchange in the electrocardiogram. Am J Cardiol 2001;88:396-9.

[8] Han C, Gregg R, Field D, Babaeizadeh S. Automatic detection of ECG cable interchange by analyzing both morphology and interlead relations. J Electrocardiol 2014; 47:781-7.

[9] Gregg R, E W Hancock, Babaeizadeh S. Detecting ECG limb lead-wire interchanges involving the right leg leadwire. Comp in Cardiol 2017;44.

[10] Jekova I, Krasteva V, Leber R, Schmid R, Twerenbold R, Müller C, Reichlin T, Abächerli R. Inter-lead correlation analysis for automated detection of cable reversals in 12/16-lead ECG. Computer Methods and Programs in Biomedicine 2016;134:31-41.

[11] Xia H, Garcia GA, Zhao X. Automatic detection of ECG electrode misplacement: a tale of two algorithms. Physiol Meas 2012;33:1549-61.

[12] Joshi KR, Morris DL, Figueredo VM. Wandering acute myocardial infarction. Am J Med. 2014;127(4):e5-e6.

[13] Givens P, Goldonowicz J, Littmann L. The electrocardiogram of chest and limb lead reversal. Am J Med. 2014;127(9):e29-e30.

Address for correspondence

Irena Jekova

Bulgarian Academy of Sciences,

Institute of Biophysics and Biomedical Engineering,

Acad. G. Bonchev str., bl.105, 1113, Sofia, Bulgaria

irena@biomed.bas.bg. 